\title{
Automatic Navigation of a Long Range Rocket Vehicle
}

\author{
H. S. TSIEN, ${ }^{1}$ T. C. ADAMSON, ${ }^{2}$ and E. L. KNUTH ${ }^{2}$
}

Daniel and Florence Guggenheim Jet Propulsion Center, California Institute of Technology, Pasadena, Calif.

\begin{abstract}
The flight of a rocket vehicle in the equatorial plane of a rotating earth is considered with possible disturbances in the atmosphere due to changes in density, in temperature, and in wind speed. These atmospherie disturbances together with possible deviations in weight and in moment of inertia of the vehicle tend to change the flight path away from the normal flight path. The paper gives the condition for the proper cut-off time for the rocket power, and the proper corrections in the elevator angle so that the vehicle will land at the chosen destination in spite of such disturbances. A scheme of tracking and automatic navigation involving a high-speed computer and elevator servo is suggested for this purpose.
\end{abstract}

$\mathbf{T}$ HE behavior of a vehicle flying through air is closely dependent upon the aerodynamic forces acting upon the vehicle. If, during one period of oscillation of the vehicle, there is appreciable variation of the response of aerodynamic forces to the attitude of the vehicle through variations in speed, in aerodynamic coefficients, in air density, etc., then the behavior of the disturbed flight path cannot be described by a linear differential equation of constant coefficients. In fact, the basic differential equation actually has coefficients that are specified functions of time. A very simple example of such motion is that of an artillery rocket during burning of the propellant grain. As shown by J. B. Rosser, R. R. Newton, and G. L. Gross (1), ${ }^{3}$ the basic differential equation for this particular case can be written as Bessel's differential equation for the order $1 / 2$. The general character of the solutions of such differential equations is quite different from the character of solutions of differential equations with constant coefficients. For instance, while for equations with constant coefficients the stability of solutions for the homogeneous equation is generally sufficient to insure the stability of solutions with reasonable forcing functions, this simple state of affairs no longer prevails for equations with variable coefficients. The present theory of control and stability is built almost exclusively upon the theory of differential equations with constant coefficients. Therefore to study the disturbed motion of rockets, new methods have to be used.

R. Drenick in a recent paper (2) demonstrated the usefulness of ballistic disturbance theory in solving the control and guidance problem of ballistic trajec-

Received April 28, 1952.

${ }^{1}$ Robert H. Goddard Professor of Jet Propulsion.

2 Daniel and Florence Guggenheim Jet Propulsion Fellows. The computations involved in this paper were carried out by R. C. Evans, Daniel and Florence Guggenheim Jet Propulsion Fellow, and F. W. Hartwig, Captain U.S.A.F., in addition to the junior authors. tories described by equations with time-varying coefficients. His theory is based upon the method of adjoint functions, first introduced by G. A. Bliss (3) during World War I. The purpose of the present paper is to make Drenick's theory more complete and definite and to apply it to the problem of automatic navigation of a long-range winged rocket vehicle. A system of control involving a fast computer is suggested, whereby the range errors due to changing atmospheric conditions and deviation from the standard weight of the vehicle are automatically corrected. The main objective here is not, however, to give the final design of such an automatic navigation system, but rather to show the power of the ballistic disturbance theory for solving such problems and the various elements necessary for such a navigational system.

\section{Equations of Motion}

In order not to complicate matters, the vehicle is assumed to move in the equatorial plane of the rotating earth (Fig. 1). The planar motion is possible due to the absence of cross Coriolis force in the equatorial plane. The co-ordinate system is fixed with respect to the rotating earth, i.e., actually it rotates with the angular velocity $\Omega$, the speed of earth rotation. The value of $\Omega$ is as follows:

$$
\Omega=7.2921 \times 10^{-5} \mathrm{rad} / \mathrm{sec} \ldots \ldots \ldots \ldots[1]
$$

In the equatorial plane, the position of the vehicle at any time instant $t$ is specified by the radius $r$ and angle $\theta$ from the starting point of the vehicle. $r_{0}$ is the mean earth radius, its value is

$$
r_{0}=20.88 \times 10^{6} \mathrm{ft} .
$$

If $g$ is the gravitational constant at the surface of the earth without the centrifugal force due to rotation, then

$$
g=32.2577 \mathrm{ft} / \mathrm{sec}^{2} \ldots \ldots \ldots \ldots \ldots \text { [3] }
$$

Let $\mathrm{R}$ and $\theta$ be the force per unit mass acting on the vehicle in the radial and the circumferential directions, respectively. Then the equations of motion of the center of gravity of the vehicle are

$$
\left.\begin{array}{rl}
\frac{d r}{d t} & =\dot{r} \\
\frac{d \theta}{d t} & =\dot{\theta} \\
\frac{d r}{d t} & =R+r(\dot{\theta} \pm \Omega)^{2}-g\left(\frac{r_{0}}{r}\right)^{2} \\
r \frac{d \dot{\theta}}{d t} & =\theta-2 \dot{r}(\dot{\theta} \pm \Omega)
\end{array}\right\}
$$

${ }^{3}$ Numbers in parentheses refer to the References on page 199. 
where the plus sign in the second terms to the right will be valid for flights toward the east, and the minus sign for flights toward the west.

The forces acting on the center of gravity of the vehicle are the thrust $f$, the lift $L$, and the $\operatorname{drag} D$ (Fig. 1). Let $W$ be the instantaneous weight of the vehicle with respect to $g$, and $V$ the magnitude of air velocity relative to the vehicle. Then it is convenient to introduce the parameters $\Psi, \Lambda$, and $\Delta$ as follows:

$$
\Psi=\frac{f g}{W}, \quad \Lambda=\frac{L g}{W V}, \quad \Delta=\frac{D g}{W V}
$$

It will be assumed that the natural wind velocity $w$ is in the horizontal direction, positive if it is a head wind. $w$ is considered as a function of altitude $r$. If $v_{r}$ is the radial velocity and $v_{\theta}$ the circumferential velocity, i.e.,

$$
\left.\begin{array}{l}
v_{r}=\dot{r} \\
v_{\theta}=r \dot{\theta}
\end{array}\right\}
$$

then relative air velocity $V$ is computed as

$$
V^{2}=v_{r}^{2}+\left(v_{\theta}+w\right)^{2} .
$$

If $\beta$ is the angle between the thrust line and the hori zontal direction, then the components of forces $\mathrm{R}$ and $\theta$ per unit mass are

$$
\left.\begin{array}{l}
R=\Psi \sin \beta+\left(v_{\theta}+w\right)_{\Lambda}-v_{r} \Delta \\
\theta=\Psi \cos \beta-v_{r} \Lambda-\left(v_{\theta}+w\right) \Delta
\end{array}\right\}
$$

If $N$ is the moment of forces about the center of gravity, divided by the moment of inertia of the vehicle, the equation for the angular acceleration is

$$
\frac{d \dot{\beta}}{d t}=\frac{d \dot{\theta}}{d t}+N
$$

To completely specify the motion of the vehicle, the lift $L$, the $\operatorname{drag} D$, and the moment $m$ about the center of gravity have to be given as functions of time. According to the aerodynamic convention, the $L$ and $D$ will be expressed in terms of the lift coefficient $C_{L}$ and the drag coefficient $C_{D}$ as follows:

$$
\left.\begin{array}{rl}
L & =\frac{1}{2} \rho V^{2} S C_{L} \\
D & =\frac{1}{2} \rho V^{2} S C_{D}
\end{array}\right\}
$$

where $\rho$ is the air density, a function of the altitude $r$, and $S$ is a fixed reference area, say the wing area of the vehicle. In the present problem, since the motion of the vehicle is restricted to the equatorial plane, the attitude of the vehicle essential for aerodynamic calculations is determined by the angle of attack $\alpha$, i.e., the angle between the thrust line, or body axis and the relative air velocity vector (Fig. 1). The control on the motion of the vehicle is affected, however, through the elevator angle $\epsilon$. The parameters which will affect $C_{L}$ and $C_{D}$ are thus $\alpha$ and $\epsilon$. In addition, the aerodynamic coefficients are functions of the Reynolds number $R e$ and the Mach number $M$. Thus

$$
\left.\begin{array}{l}
\left.C_{L}=C_{L}(\alpha, \epsilon, M, R e)\right) \\
\left.C_{D}=C_{D}(\alpha, \epsilon, M, R e)\right\}
\end{array}\right\}
$$

\footnotetext{
${ }^{4}$ Drenick (Ref. 2) seems to take the sense of the angle of attack $\alpha$ opposite to the convention of aerodynamicists.
}

It will be assumed that the thrust line passes through the center of gravity of the vehicle; thus the thrust gives no moment. Since the angular motion of the vehicle during the powered flight is expected to be slow, the jet damping moment of the rocket is negligible. The only moment acting on the vehicle is then the aerodynamic moment $m$. $m$ can be also expressed as a coefficient $C_{M}$ as follows:

$$
m=\frac{1}{2} \rho V^{2} S l C_{M}
$$

where $l$ is a reference length. The moment coefficient $C_{M}$ is again a function of the four parameters $\alpha, \epsilon, M$, and $R e$, or

$$
C_{M}=C_{M}(\alpha, \epsilon, M, R e)
$$

If $I$ is the moment of inertia of the vehicle, then the magnitude of $N$ in Equation [9] is

$$
N=m / I
$$

With the rotations defined above, the system of equations of motion is as follows:

$$
\left.\begin{array}{c}
\frac{d r}{d t}=v_{r} \\
\frac{d \theta}{d t}=v_{\theta} / r \\
\frac{d \beta}{d t}=\dot{\beta} \\
\frac{d v_{r}}{d t}=\Psi \sin \beta+\left(v_{\theta}+w\right) \Lambda-v_{r} \Delta+r\left(\frac{v_{\theta}}{r} \pm \Omega\right)^{2}- \\
g\left(\frac{r_{0}}{r}\right)^{2}=F \\
\frac{d v_{\theta}}{d t}=\Psi \cos \beta-v_{r} \Lambda-\left(v_{\theta}+w\right)_{\Delta}-2 v_{r}\left(\frac{v_{\theta}}{r}+\Omega\right)+ \\
\frac{v_{\theta} v_{r}}{r}=G \\
\frac{d \dot{\beta}}{d t}=\frac{1}{r}\left\{\Psi \cos \beta-v_{r} \Lambda-\left(v_{\theta}+w\right) \Delta\right\}-\quad \\
2 \frac{v_{r}}{r}\left(\frac{v_{\theta}}{r}+\Omega\right)+N=H
\end{array}\right\}
$$

This system of equations is a set of first order equations for the six unknowns $r, \theta, \beta, v_{\tau}, v_{\theta}$, and $\dot{\beta}$. To solve it,

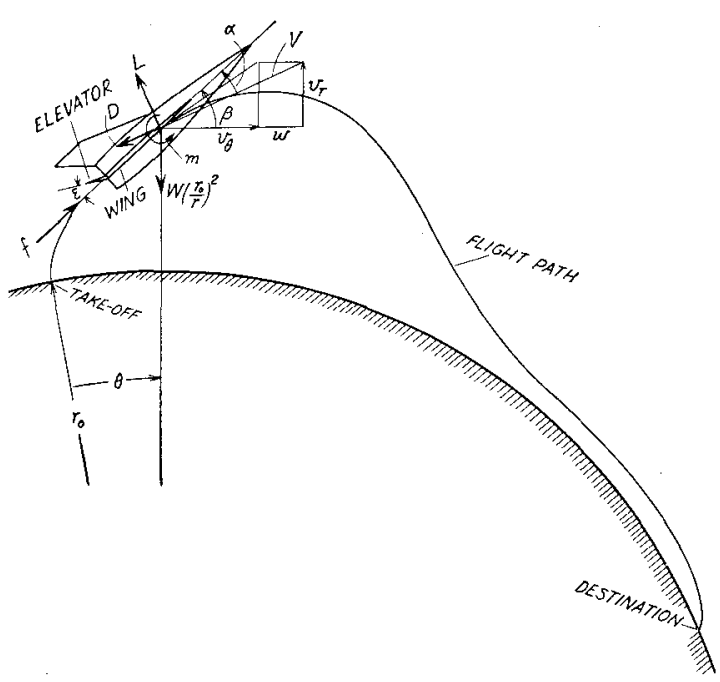

FIG. 1 FLIGHT PATH OF A LONG RANGE WINGED ROCKET (The size of the vehicle is greatly magnified for clear graphical representation.) 
the six initial values at the start, $t=0$, for the unknowns must be specified. In addition, the thrust $f$, the weight $W$, the moment of inertia $I$ must be given for every time instant. To determine the aerodynamic forces, the elevator angle $\epsilon$ must be specified as a function of time. The properties of the atmosphere must be known; i.e., wind velocity $w$, air density $\rho$, air viscosity and air velocity of sound must be given as functions of the altitude $r$. The angle of attack $\alpha$ of the vehicle cannot be specified; it is a quantity to be computed from the angle $\beta$ and the relative air velocity vector $V$.

\section{Normal Flight Path}

Let the properties of the atmosphere be standardized and known as the properties of the normal atmosphere. The average characteristics of the vehicle and its power plant can be taken to be representative. Then if the elevator angle $\epsilon$ is given as a function of time, the flight path of the vehicle is determined and can be calculated by integrating the system of Equation [15]. The actual execution of this computation will be probably done on an electro-mechanical computer. This flight path of a standardized vehicle in normal atmosphere can be called the normal flight path.

The dominating feature of the normal flight path is its range. This range is the distance between the takeoff point and the landing point. The problem of navigation is then to calculate the proper time for cut-off of the rocket and the proper variation of the elevator angle during flight so that the range is that desired. This problem of navigation for the standardized vehicle in normal atmosphere can be solved mathematically before the actual take-off of the vehicle, since all information for the normal flight path is known or specified beforehand.

\section{Disturbance Equations}

Natural atmospheric characteristics do not, of course, coincide with those assumed for the atmosphere. The wind velocity at each altitude changes according to the weather conditions; the temperature $T$ is also a varying quantity. Therefore one should expect variations from the normal flight path due to changes in atmospheric conditions. The actual vehicle also may be somewhat different from the standardized vehicle; in weight, rocket performance, etc. Therefore actual flight path will be different from the normal flight path if the same elevator angle programming is used. The problem of navigation of an actual vehicle is that of correcting the elevator angle programming so that the range of the actual flight will be the same as the normal flight path and the destination is reached without error. Due to the rapidity of flight, this navigational problem cannot be solved by conventional method; but should be solved by an automatic computing system, which responds to every deviation from the normal conditions with a speed approaching instant action.

The general problem of automatic navigation is very difficult indeed. However, the deviations from the normal conditions are expected to be small, since the normal flight path is, after all, is good representation of the average situation. This fact immediately suggests that only first order quantities in deviations need be considered. This "linearization" is the basis of the ballistic disturbance theory and the present theory of automatic navigation.

Let quantities of the normal flight path be denoted by a bar over them and deviations by the $\delta$ sign. Thus for the actual flight path,

$$
\begin{aligned}
& r=\bar{r}+\delta r, \quad \theta=\bar{\theta}+\delta \theta, \quad \beta=\bar{\beta}+\delta \beta\rangle \\
& \left.v_{r}=\bar{v}_{r}+\delta v_{r}, \quad v_{\theta}=\bar{v}_{\theta}+\delta v_{\theta}, \quad \dot{\beta}=\bar{\beta}+\delta \dot{\beta}\right\}
\end{aligned}
$$

The deviations of the actual atmosphere from the normal atmosphere are expressed as the deviation of density $\delta \rho$, the deviation of temperature $\delta T$, and the deviation of wind velocity $\delta w$; thus

$$
\rho=\bar{\rho}+\delta \rho, \quad T=\bar{T}+\delta T, \quad w=\bar{w}+\delta w
$$

The deviation of the actual vehicle from the normal vehicle is assumed to be limited only to deviation of weight $\delta W$ and the moment of inertia $\delta I$. That is

$$
W=\bar{W}+\delta W, \quad I=\bar{I}+\delta I .
$$

The rate of propellant flow and the effective exhaust velocity of the rocket is assumed to be standard. The wing area $S$ and the aerodynamic characteristies of the vehicle as expressed by Equations [11] and [13] are also assumed to be invariant.

By substituting Equations [16], [17], and [18] into the equations of motion, Equation [15], and retaining only first term deviations, one has

$$
\begin{aligned}
& \frac{d \delta r}{d t}=+\delta v_{r} \\
& \frac{d \delta \theta}{d t}=-\frac{\bar{v}_{\theta}}{\bar{r}^{2}} \delta r+\frac{1}{\bar{r}^{2}} \delta v_{\theta} \\
& \frac{d \delta \beta}{d t}=+\delta \dot{\beta}
\end{aligned}
$$

$$
\left.\begin{array}{c}
\frac{d \delta v_{r}}{d t}=a_{1} \delta r+a_{2} \delta \beta+a_{3} \delta v_{r}+a_{4} \delta v_{\theta}+a_{5} \delta \epsilon+ \\
a_{6} \delta \rho+a_{7} \delta T+a_{8} \delta w+a_{9} \delta W \\
\frac{d \delta v_{\theta}}{d t}=b_{1} \delta r+b_{9} \delta B \beta+b_{3} \delta v_{r}+b_{4} \delta v_{\theta}+b_{5} \delta \epsilon+ \\
b_{6} \delta \rho+b_{7} \delta T+b_{8} \delta w+b_{9} \delta W \\
\frac{d \delta \dot{\beta}}{d t}=c_{1} \delta r+c_{2} \delta \beta+c_{8} \delta v_{r}+c_{4} \delta v_{\theta}+c_{5} \delta \epsilon+ \\
c_{6} \delta \rho+c_{7} \delta T+c_{8} \delta w+c_{9} \delta W+c_{10} \delta I
\end{array}\right\}
$$

The coefficients $a$ 's, $b$ 's, and c's are partial derivatives of $F, G$, and $H$ defined by Fquations [15], evaluated on the normal flight path. That is, for example,

$$
\begin{aligned}
& a_{1}=\left(\frac{\overline{\partial F}}{\partial r}\right), \quad a_{2}=\left(\frac{\overline{\partial F}}{\partial \beta}\right), \quad a_{3}=\left(\frac{\overline{\partial F}}{\partial v_{r}}\right), \\
& a_{4}=\left(\frac{\overline{\partial F}}{\partial v_{\theta}}\right), \quad a_{5}=\left(\frac{\overline{\partial F}}{\partial \epsilon}\right), \quad a_{6}=\left(\frac{\partial F}{\partial \rho}\right), \\
& a_{7}=\left(\frac{\overline{\partial F}}{\partial T}\right), \quad a_{8}=\left(\overline{\frac{\partial F}{\partial w}}\right), \quad a_{9}=\left(\overline{\frac{\partial F}{\partial W}}\right)
\end{aligned}
$$

These coefficients are calculated in detail and given in the Appendix.

The system of Equations [19] and [20] is the system of disturbance equations. They are linear. The coefficients when evaluated on the normal flight path are 
finally specified functions of time. If the deviations of the atmospheric properties $\delta \rho, \delta T$, and $\delta w$ are known, and if $\delta \epsilon, \delta W$, and $\delta I$ are specified, then this system of differential equations determines the deviations $\delta r, \delta \theta$, $\delta \beta, \delta v_{r}, \delta v_{\theta}$, and $\delta \dot{\beta}$ from the normal trajectory. This is the direct problem in the ballistic disturbance theory. The problem of automatic navigation is however different from this. What is required is the function $\delta \epsilon$, correction to the elevator angle, such that the range error is zero. As suggested by Drenick (2), this navigational problem can best be solved by the method of adjoint functions of Bliss.

\section{Adjoint Functions for Range Correction}

The principle of the method of adjoint functions is as follows (3): Let $y_{i}(t), i=1, \ldots n$, be determined by a system of linear equations

$$
\frac{d y_{i}}{d t}=\sum_{j=1}^{n} a_{i j} y_{j}+Y_{i} \ldots \ldots \ldots \ldots \ldots
$$

where $a_{i j}$ are given coefficients which may be functions of the time $t$, and $Y_{i}$ are specified functions of time. Now introduce a new set of functions $\lambda_{i}(t)$, called the adjoint functions to $y_{i}(t)$, which satisfy the following system of differential equations

$$
\frac{d \lambda_{i}}{d t}=-\sum_{j=1}^{n} a_{j i} \lambda_{j} \ldots \ldots \ldots \ldots \ldots
$$

By multiplying Equation [22] by $\lambda_{i}$ and Equation [23] by $y_{i}$, and then adding the resultant equations, it can be shown that

$$
\frac{d}{d t} \sum_{i=1}^{n} \lambda_{i} y_{i}=\sum_{i=1}^{n} \lambda_{i} Y_{i} \ldots \ldots \ldots \ldots \ldots
$$

Equation [24] can be integrated from $t=t_{1}$ to $t=t_{2}$,

$$
\left.\sum_{i=1}^{n} \lambda_{i} y_{i}\right|_{t=t_{2}}=\left.\sum_{i=1}^{n} \lambda_{i} y_{i}\right|_{t=t_{1}}+\int_{t=t_{1}}^{t=t_{2}}\left(\sum_{i=1}^{n} \lambda_{i} Y_{i}\right) d t .
$$

Bliss named Equation [25] the "Fundamental Formula."

For the present problem, the $y_{i}$ are the disturbance quantities, i.e.,

$$
\left.\begin{array}{lll}
y_{1}=\delta r, & y_{2}=\delta \theta, & y_{3}=\delta \beta \\
y_{4}=\delta v_{r}, & y_{5}=\delta v_{\theta}, & y_{6}=\delta \beta
\end{array}\right\}
$$

Then, according to Equation [20], the adjoint functions $\lambda_{i}$ satisfy the following differential equations

$$
\begin{aligned}
& -\frac{d \lambda_{1}}{d \bar{t}}=-\frac{\bar{v}_{\theta}}{\bar{r}^{2}} \lambda_{2} \quad+a_{1} \lambda_{4}+b_{1} \lambda_{5}+c_{1} \lambda_{6} \\
& -\frac{d \lambda_{2}}{d t}=0 \\
& -\frac{d \lambda_{3}}{d \bar{t}}= \\
& -\frac{d \lambda_{4}}{d t}=\lambda_{1} \\
& -\frac{d \lambda_{5}}{d t}=\quad \frac{1}{\bar{r}} \lambda_{2} \\
& -\frac{d \lambda_{6}}{d t}= \\
& \left.\begin{array}{r}
a_{2} \lambda_{4}+b_{2} \lambda_{5}+c_{2} \lambda_{6} \\
+a_{3} \lambda_{4}+b_{3} \lambda_{5}+c_{3} \lambda_{6} \\
+a_{4} \lambda_{4}+b_{4} \lambda_{5}+c_{4} \lambda_{6}
\end{array}\right\}
\end{aligned}
$$

$$
\left.\begin{array}{l}
Y_{1}=Y_{2}=Y_{3}=0 \\
Y_{4}=a_{5} \delta \epsilon+a_{6} \delta \rho+a_{7} \delta T+a_{8} \delta w+a_{9} \delta W \\
Y_{5}=b_{5} \delta \epsilon+b_{6} \delta \rho+b_{7} \delta T+b_{8} \delta w+b_{9} \delta W \\
Y_{6}=c_{5} \delta \epsilon+c_{6} \delta \rho+c_{7} \delta T+c_{8} \delta w+c_{9} \delta W+c_{10} \delta I
\end{array}\right\}
$$

The Equations [27] do not determine the $\lambda$-functions completely. To do that, a set of values for $\lambda$ must be specified at a certain instant. For the problem of automatic navigation, the requirement is vanishing range error; then the required boundary conditions for Equations [27] can be determined as follows: If $t_{2}$ is the time instant of landing of the actual vehicle, $\bar{t}_{2}$ the time instant of landing of the normal flight path, then

$$
t_{2}=t_{2}+\delta t_{2} \ldots \ldots \ldots \ldots \ldots \ldots[29]
$$

Similarly with the subscript 2 denoting the quantities at the landing instant

$$
\left.\begin{array}{l}
r_{2}=\bar{r}_{2}+\delta r_{2} \\
\theta_{2}=\bar{\theta}_{2}+\delta \theta_{2}
\end{array}\right\}
$$

But

$$
\left.\begin{array}{l}
\delta r_{2}=\left(\bar{v}_{r}\right)_{t=\bar{t}_{2}} \delta t_{2}+(\delta r)_{t=\bar{t}_{2}} \\
\delta \theta_{2}=\frac{1}{r_{0}}\left(\bar{v}_{\theta}\right)_{t=\bar{t}_{2}} \delta t_{2}+(\delta \theta)_{t=\bar{t}_{2}}
\end{array}\right\} \cdots \cdots \cdots \cdots
$$

$\delta r_{2}$ is, however, zero by definition, because landing means contact with the surface of earth, $\bar{r}_{2}=r_{2}=r_{0}$. By eliminating $\delta t_{2}$ from the Equations [31],

$$
\delta \theta_{2}=\left[-\frac{1}{\bar{r}}\left(\frac{\bar{v}_{\theta}}{\bar{v}_{r}}\right) \delta r+\delta \theta\right]_{t=\bar{t}_{2}} \cdot
$$

Therefore if the magnitudes of $\lambda_{i}$ at the landing instant $t=\bar{t}_{2}$ are specified as

$$
\left.\begin{array}{l}
\lambda_{1}=-\frac{1}{\bar{r}}\left(\frac{\bar{v}_{\theta}}{\bar{v}_{r}}\right) \\
\lambda_{2}=1 \\
\lambda_{3}=\lambda_{4}=\lambda_{5}=\lambda_{6}=0
\end{array}\right\}
$$

then the error in range is given by

$$
\begin{aligned}
& \delta \theta_{2}=\left.\sum_{i=1}^{n} \lambda_{i} y_{i}\right|_{t=\bar{t}_{2}}= \\
& \left.\left[\lambda_{1} \delta r+\lambda_{2} \delta \theta+\lambda_{3} \delta \beta+\lambda_{4} \delta v_{r}+\lambda_{\delta} \delta v_{\theta}+\lambda_{6} \delta \dot{\beta}\right]\right]_{t=\bar{t}_{2} .} .
\end{aligned}
$$

When the normal flight path is determined, the coefficients in Equation [27] are specified as functions of time. These equations together with the end conditions of Equation [33] then determine the adjoint functions $\lambda_{i}$. The integration has to be performed "backwards" for $t<\bar{t}_{2}$, by perhaps an electro-mechanical computer. With the adjoint functions so determined, one can use the Fundamental Formula of Equation [25] to modify the equation for the range error given by Equation [34]: Let $\bar{t}_{1}$ denote the time instant for the power cut-off. Then the condition for the error $\delta \theta_{2}$ in range to be zero can be expressed as

$$
\left.\begin{array}{rl}
\delta \theta_{2} & =0=\left[\lambda_{1} \delta r+\lambda_{2} \delta \theta+\lambda_{3} \delta \beta+\lambda_{4} \delta v_{r}+\lambda_{5} \delta v_{\theta}+\lambda_{6} \delta \dot{\beta}\right] t=t_{1} \\
& +\int_{t_{1}}^{\bar{t}_{2}}\left[\lambda_{4} Y_{4}+\lambda_{5} Y_{5}+\lambda_{6} Y_{6}\right] d t
\end{array}\right\}
$$

This is the basic equation for automatic navigation. It will be exploited presently.

\section{Cut-Off Condition}

The condition of Equation [35] for arbitrary dis-

The $Y_{i}$ are then 
turbances can be broken down into two parts: The sum and the integral to be set to zero separately. Therefore the condition to be satisfied at the normal cut-off instant $\bar{t}_{1}$ is

$$
\left[\lambda_{1} \delta r+\lambda_{2} \delta \theta+\lambda_{3} \delta \beta+\lambda_{4} \delta v_{r}+\lambda_{i} \delta v_{\theta}+\lambda_{6} \delta \dot{\beta}\right]_{t=\bar{t}_{1}=0 .}
$$

Since the normal cut-off instant $\bar{t}_{1}$ is a standard time instant, but not necessarily the actual cut-off instant $t_{1}$, i.e.,

$$
t_{1}=t_{1}+\delta t_{1} \ldots
$$

Equation [36] should be converted into a more useful form involving the quantities at the actual cut-off instant. This is easily done, because up to the first order quantities, according to Equation [16]

$$
(\delta r)_{t=\bar{t}_{1}}=(r)_{t=t_{1}}-\left(\frac{\overline{d r}}{d t}\right)_{t=\bar{t}_{1}} \delta t_{1}-(\bar{r})_{t=\bar{t}_{1}}
$$

Or

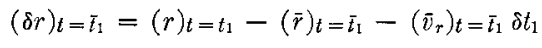

Similarly,

$$
\begin{aligned}
& (\delta \theta)_{t=\bar{t}_{1}}=(\theta)_{t=t_{1}}-(\bar{\theta})_{t=\bar{t}_{1}}-\left(\frac{1}{\bar{r}} \bar{v}_{\theta}\right)_{t=\bar{t}_{1}} \delta t_{1} \\
& (\delta \beta)_{t=\bar{t}_{1}}=(\beta)_{t=t_{1}}-(\bar{\beta})_{t=\bar{t}_{1}}-(\dot{\beta})_{t=\bar{t}_{1}} \delta t_{1}
\end{aligned}
$$

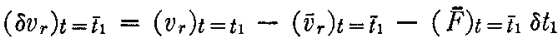

$$
\begin{aligned}
& \left(\delta v_{\theta}\right)_{t}=\bar{t}_{1}=\left(v_{\theta}\right)_{t=t_{1}}-(\bar{v} \theta)_{t}=\bar{t}_{1}-(\bar{G})_{t=\bar{t}_{1}} \delta t_{1} \\
& (\delta \dot{\beta})_{t=\bar{t}_{1}}=(\dot{\beta})_{t=t_{1}}-(\overline{\dot{\beta}})_{t=\bar{t}_{1}}-(\bar{H})_{t=\bar{t}_{1}} \delta t_{1}
\end{aligned}
$$

where $\bar{F}, \bar{G}$, and $\bar{H}$, are the values of these quantities given by Equation [15] evaluated on the normal flight path. In fact, they should be evaluated at an instant just before the normal cut-off time $\bar{t}_{1}$ so that the accelerating force of the rocket is included and the rates of change of velocities are those of a powered flight. Now define $J$ and $\bar{J}$ as follows,

$$
J=\left[\lambda_{1}{ }^{*} r+\lambda_{2}{ }^{*} \theta+\lambda_{3}{ }^{*} \beta+\lambda_{4}{ }^{*} v_{r}+\lambda_{5}{ }^{*} v_{\theta}+\lambda_{6}{ }^{*} \dot{\beta}\right] t=t_{1}
$$

and

$$
\bar{J}=\left[\lambda_{1}{ }^{*} \bar{r}+\lambda_{2}{ }^{*} \bar{\theta}+\lambda_{3}{ }^{*} \bar{\beta}+\lambda_{4}{ }^{*} \bar{v}_{r}+\lambda_{5}{ }^{*} v_{\theta}+\lambda_{6}{ }^{*} \dot{\beta}\right] t=\overline{1}_{1}
$$

where $\lambda_{i}{ }^{*}$ are the values of $\lambda_{i}$ evaluated at the normal cut-off time $t_{1}$. Then the condition to be satisfied at the actual cut-off instant $t_{1}$ is

$$
\begin{array}{r}
J=\bar{J}+\left\{\lambda_{1}{ }^{*} \bar{v}_{r}+\lambda_{2} * \frac{\bar{v}_{\theta}}{\bar{r}}+\lambda_{3}{ }^{*} \dot{\beta}+\lambda_{4} * \bar{F}+\lambda_{5}{ }^{*} G+\lambda_{6} * \bar{H}\right\}_{t=t_{1}} \\
\left(t_{1}-\bar{t}_{1}\right) \ldots[39]
\end{array}
$$

This is the equation to determine the proper instant of power cut-off.

When the normal flight path is known, $\vec{J}$ and the quantity within the bracket to the right of Equation [39] are fixed. Then the whole right-hand side of Equation [39] can be considered as a linearly increasing function of time $t$, if $t$ is substituted for $t_{1}$. Simultaneously $J$ can be computed at every instant before cut-off by using the predetermined $\lambda_{i}{ }^{*}$ and values of position and velocity of the actual vehicle obtained by tracking stations. The magnitudes of the quantities on the two sides of Equation [39] can then be continuously compared. When they are equal to each other, condition $[39]$ is satisfied. Then the power cut-off signal is given and the rocket power is shut off. ${ }^{5}$

\section{Condition for Automatic Navigation}

When the rocket power is shut off earlier or later than the normal cut-off instant $\bar{t}_{1}$, the propellant left in the tank, if not dumped, will alter the weight $W$ and the moment of inertia $I$ of the vehicle. It is also possible that the pay load of the vehicle is not that specified for the standard vehicle. Then after power-off, there is a fixed $\delta W$ and $\delta I$, fixed in the sense that they do not change with time, and are known once the power cut-off is affected. Of different character are the deviations $\delta \rho, \delta T, \delta w$ of the actual atmosphere from the standard atmosphere. These are not known unless they are measured. In the following, it is proposed to use the vehicle itself as a measuring instrument, and proceed as follows:

After the cut-off condition is satisfied, the condition for zero range error is that the integral in Equation [35] should vanish. Now since the $Y_{i}$ in that integrand involving arbitrary disturbances $\delta \rho, \delta T$, and $\delta w$ is not known beforehand, this condition can be satisfied only if the integrand itself vanishes. That is, according to Equations [28],

$\left(\lambda_{4} a_{5}+\lambda_{5} b_{5}+\lambda_{6} c_{5}\right) \delta \epsilon+\left(\lambda_{4} a_{6}+\lambda_{5} b_{6}+\lambda_{6} c_{6}\right) \delta \rho+$

$\left(\lambda_{4} a_{7}+\lambda_{5} b_{7}+\lambda_{6} c_{7}\right) \delta T+\left(\lambda_{4} a_{8}+\lambda_{5} b_{8}+\lambda_{6} c_{8}\right) \delta w+\left(\lambda_{4} a_{9}+\right.$ $\left.\lambda_{5} b_{9}+\lambda_{6} c_{9}\right) \delta W+\lambda_{6} c_{10} \delta I=0$

Or, with the following notation

$$
\left.\begin{array}{l}
d_{5}=\lambda_{4} a_{5}+\lambda_{5} b_{5}+\lambda_{6} c_{5} \\
d_{6}=\lambda_{4} a_{6}+\lambda_{5} b_{6}+\lambda_{6} c_{6} \\
d_{7}=\lambda_{4} a_{7}+\lambda_{5} b_{7}+\lambda_{6} c_{7} \\
d_{8}=\lambda_{4} a_{8}+\lambda_{5} b_{8}+\lambda_{6} c_{8} \\
D=-\left(\lambda_{4} a_{9}+\lambda_{5} b_{9}+\lambda_{6} c_{9}\right) \delta W-\lambda_{6} c_{10} \delta I
\end{array}\right\}
$$

this condition can be written as

$$
d_{5} \delta \epsilon+d_{6} \delta \rho+d_{7} \delta T+d_{8} \delta w=D \ldots
$$

Equation [20] can be rewritten as

$$
\left.\begin{array}{l}
a_{5} \delta \epsilon+a_{6} \delta \rho+a_{7} \delta T+a_{8} \delta w=A \\
b_{5} \delta \epsilon+b_{8} \delta \rho+b_{7} \delta T+b_{8} \delta w=B \\
c_{5} \delta \epsilon+c_{6} \delta \rho+c_{7} \delta T+c_{8} \delta w=C
\end{array}\right\}
$$

where

$$
\left.\begin{array}{l}
A=\frac{d \delta v_{r}}{d t}-a_{1} \delta r-a_{2} \delta \beta-a_{3} \delta v_{r}-a_{4} \delta v_{\theta}-a_{7} \delta W \\
B=\frac{d \delta v_{\theta}}{d t}-b_{1} \delta r-b_{2} \delta \beta-b_{3} \delta v_{r}-b_{4} \delta v_{\theta}-b_{7} \delta W \\
C=\frac{d \delta \dot{\beta}}{d t}-c_{1} \delta r-c_{2} \delta \beta-c_{3} \delta v_{r}-c_{4} \delta v_{\theta}-c_{9} \delta W-c_{10} \delta I
\end{array}\right\}
$$

If the tracking stations for the vehicle will measure the quantities $A, B$, and $C$, then the atmospheric disturbances $\delta \rho, \delta T$, and $\delta w$ can be determined by solving for these variations using Equation [42]. This is essentially using the vehicle itself as a measuring instrument for $\delta \rho, \delta T$, and $\delta w$. When $\delta \rho, \delta T$, and $\delta w$ are known, Equation [41] gives the proper elevator angle correction $\delta \epsilon$.

A mathematically equivalent way to calculate $\delta \epsilon$

'Drenick's cut-off condition (Ref. 2) differs from that of Equation [39] in that the second term to the right is not present. It seems that this neglect is not justified and will introduce first order range error. 
would be to directly solve for $\delta \epsilon$ using the system of Equations [41] and [42]. Thus

$$
\left|\begin{array}{cccc}
a_{5} & a_{6} & a_{7} & a_{8} \\
b_{5} & b_{6} & b_{7} & b_{8} \\
c_{5} & c_{6} & c_{7} & c_{8} \\
d_{5} & d_{6} & d_{7} & d_{8}
\end{array}\right| \delta \epsilon=\left|\begin{array}{cccc}
A & a_{6} & a_{7} & a_{8} \\
B & b_{6} & b_{7} & b_{8} \\
C & c_{6} & c_{7} & c_{8} \\
D & d_{6} & d_{7} & d_{8}
\end{array}\right| \ldots[44]
$$

This equation specifies the necessary change in the elevator angle at every instant to be calculated from the quantities $a$ 's, $b$ 's, $c$ 's, and $A, B, C, D$ at the same instant. These quantities consist partly of predetermined information from the normal flight path, and partly of measured information on the position and the velocities of vehicle obtained by tracking the vehicle. At high altitudes where the air density is very small, the aerodynamic forces will be almost negligible in comparison with the gravitational and inertia forces. Then the quantities $A, B$, and $C$ of Equation [43] will be the small difference of large magnitudes. These are then the quantities most difficult to determine accurately. If the actual elevator angle is made to conform with the one calculated by Equation [44], then in conjunction with the proper power cut-off as specified in the last section the vehicle will be navigated to the chosen landing point in spite of the atmospheric disturbances.

\section{Discussion}

When the general character of the flight path is chosen from the over-all engineering considerations, the first step is the calculation of the normal flight path using the properties of the standard atmosphere and the expected performance of the vehicle with normal weight. The knowledge of the normal flight path then determines the $a$ 's, $b$ 's, and $c$ 's. The Equation [27] together with the end conditions of Equation [33] allows the calculation of the adjoint functions $\lambda_{i}$. All this information should be on hand before the actual flight of the vehicle, and may be called the "stored data."

Before the power cut-off, the elevator angle may be programmed according to that for the normal flight path, and the stability of the vehicle is supplied by the jet vanes or by the auxiliary rockets. The tracking stations, however, go immediately into action and supply the vehicle with information on its positions and velocities. This information goes first into the cutoff computer which, using the stored information, continuously compares the magnitudes of quantities on the two sides of Equation [39], the cut-off condition. When that condition is satisfied, the power cut-off is affected.

At the instant of power cut-off, the tracking information is switched to the computer for automatic navigation. The instant of power cut-off also fixes the amount of propellant in the tank and thus determines the variations of weight $\delta W$ and inertia moment $\delta I$ from the standard. This information together with the stored data on the normal flight path then allows the computer to generate the elevator correction angle $\delta \epsilon$ according to Equations [40], [43], and [44]. Theoretically the value of $\delta \epsilon$ must be obtained without time delay from the instant when the information is received, because Equation [44] is a condition of equality of two quantities evaluated at identical time instants. Practically there will be time delay due to the finite computing time. However, it is now clear that this computing time must be made very short in order to satisfy the condition of automatic navigation as accurately as possible. The computed correction $\delta \epsilon$ combined with the elevator angle $\bar{\epsilon}$ determined for the normal flight path then gives the actual elevator angle setting $\epsilon$. The design of the control mechanism for the elevator from here on follows the conventional feed-back servomechanism, with the usual criteria of quick action, stability, and accuracy. The general scheme of the automatic navigation of the vehicle then can be represented by the sketch in Fig. 2 .

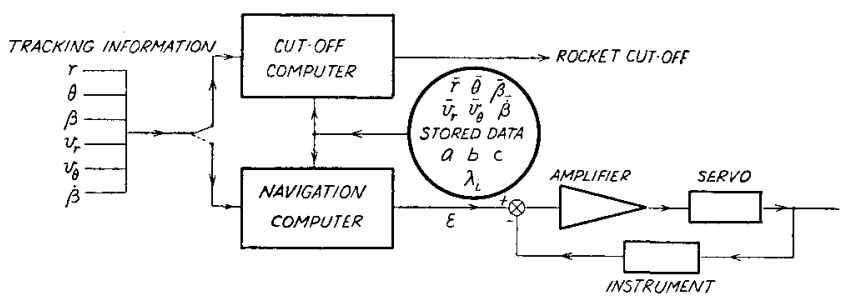

FIG. 2. SYSTEM OF AUTOMATIC NAVIGATION

The primary purpose or the "duty" of the computer is then to properly digest the dynamic and the aerodynamic information of the vehicle and thus to decide the right flight path correction that will insure landing at the chosen destination. The purpose or the duty of the elevator servo is now simply to follow the command of the computer. If the servo is considered to be internal to the vehicle, then the computer can be considered to be a mechanism to account for the external conditions of the vehicle. This separation of the internal from the external is not necessary for the control of conventional aircraft. For conventional aircraft, the basic disturbance dynamic equations have constant coefficients and the computer can be merged with the servo through a single "amplifier" of essentially RCcircuits.

The computers envisaged here are carried in the vehicle and receive the information on positions and velocities from the fixed ground tracking stations (4). It will be beyond the scope of the present paper to discuss their design. But the required accuracy and speed to generate proper signal almost instantly would indicate that they should be of the electronic digital type. If this is indeed the proper type to be used, then the separation of cut-off computer and the navigation computer is not necessary. All could be done in one computer by proper programming.

\section{Appendix}

\section{Calculation of Coefficients}

The quantities $F, G$, and $H$ are defined by Equation [15]. They contain the parameters $\Psi, \Lambda, \Delta$, and $N$. 
According to their definition as given by Equations [5] and [14], they can be written as follows:

$$
\left.\begin{array}{l}
\Psi=\frac{f g}{W} \\
\Lambda=\frac{g}{W} \frac{1}{2} \rho S C_{L} \sqrt{v_{r^{2}}+\left(v_{\theta}+w\right)^{2}} \\
\Delta=\frac{g}{W} \frac{1}{2} \rho S C_{D} \sqrt{v_{r}^{2}+\left(v_{\theta}+w\right)^{2}} \\
N=\frac{1}{I} \frac{1}{2} \rho S C_{M}\left\{v_{r}^{2}+\left(v_{\theta}+w\right)^{2}\right\}
\end{array}\right\}
$$

where the aerodynamic coefficients $C_{L}, C_{D}, C_{M}$ are functions of angle of attack $\alpha$, elevator angle $\epsilon$, Mach number $M$, and the Reynolds number Re. These aerodynamic parameters are related to quantities immediately connected with the flight path as follows:

$\alpha=\beta-\tan ^{-1} \frac{v_{r}}{v_{\theta}+w}, \quad M=V / \alpha(r), \quad$ Re $=\rho V l / \mu(r)$

where $a(r)$ is the sound velocity in atmosphere, and $\mu(r)$ is the coefficient of viscosity of air, both functions of altitude $r$. In the following calculation, the thrust $f$ will be considered to be a function of altitude only. It is also assumed that the composition of atmosphere at different altitudes remains the same as that of the standard atmosphere, only the density $\rho$ and the temperature $T$ change. Thus the variations of $a$ and $\mu$ at any altitude are variations due to temperature $T$.

For $\Psi$

$$
\frac{\partial \Psi}{\partial r}=\frac{g}{W} \frac{\partial f}{\partial r}, \quad \frac{\partial \Psi}{\partial W}=-\frac{\Psi}{W}
$$

All other partial derivatives are zero.

For $\Lambda$

$$
\begin{aligned}
& \frac{\partial \Lambda}{\partial r}=\Lambda\left[\frac{1}{\rho} \frac{d \rho}{d r}\left\{1+\frac{R e}{C_{L}} \frac{\partial C_{L}}{\partial R e}\right\}+\frac{1}{V^{2}} \frac{d w}{d r} \times\right. \\
& \left\{\left(\frac{M}{\bar{C}_{L}} \frac{\partial C_{L}}{\partial M}+\frac{R e}{C_{L}} \partial C_{L}+1\right) \times\right. \\
& \left.\left(v_{\theta}+w\right)+\frac{1}{C_{L}} \cdot \frac{\partial C_{L}}{\partial \alpha} v_{r}\right\}- \\
& \left.\frac{M}{C_{L}} \frac{\partial C_{L}}{\partial M} \frac{1}{a} \frac{d a}{d r}-\frac{R e}{C_{L}} \frac{\partial C_{L}}{\partial R e} \frac{1}{\mu} \frac{d \mu}{d r}\right] \\
& \frac{\partial \Lambda}{\partial v_{r}}=\Lambda \frac{v_{r}}{V^{2}}\left[\frac{M}{C_{L}} \frac{\partial C_{L}}{\partial M}+\frac{R e}{C_{L}} \frac{\partial C_{L}}{\partial R e}+\right. \\
& \left.1-\frac{1}{C_{L}} \frac{\partial C_{L}}{\partial \alpha} \frac{v_{\theta}+w}{v_{r}}\right] \\
& \frac{\partial \Lambda}{\partial v_{\theta}}=\Lambda \frac{v_{\theta}+w}{V^{2}}\left[\frac{M}{C_{L}} \frac{\partial C_{L}}{\partial M}+\frac{R e}{C_{L}} \frac{\partial C_{L}}{\partial R e}+\right. \\
& \frac{\partial \Lambda}{\partial \beta}=\Lambda \frac{1}{C_{L}} \frac{\partial C_{L}}{\partial \alpha} \\
& \frac{\partial \Lambda}{\partial \epsilon}=\Lambda \frac{1}{C_{L}} \frac{\partial C_{L}}{\partial \epsilon} \\
& \frac{\partial \Lambda}{\partial \rho}=\Lambda \frac{1}{\rho}\left[1+\frac{R e}{C_{L}} \frac{\partial C_{L}}{\partial R e}\right] \\
& \frac{\partial \Lambda}{\partial T}=-\Lambda\left[\frac{M}{C_{L}} \frac{\partial C_{L}}{\partial M} \frac{1}{2 T}+\frac{R e}{C_{L}} \frac{\partial C_{L}}{\partial R e} \frac{1}{\mu} \frac{\partial \mu}{\partial T}\right] \\
& \frac{\partial \Lambda}{\partial w}=\Lambda \frac{v_{\theta}+w}{V^{2}}\left[\frac{M}{C_{L}} \frac{\partial C_{L}}{\partial M}+\frac{R e}{C_{L}} \frac{\partial C_{L}}{\partial R e}+\right. \\
& \left.1+\frac{1}{C_{L}} \frac{\partial C_{L}}{\partial \alpha} \frac{v_{r}}{v_{\theta}+w}\right]=\frac{\partial \Lambda}{\partial v_{\theta}} \\
& 1+\frac{1}{C_{L}} \frac{\partial C_{L}}{\partial \alpha} \frac{v_{r}}{v_{\theta}+w}
\end{aligned}
$$

For $\Delta$, the partial derivatives are obtained from the above equations by substituting $\Delta$ for $\Lambda$, and $C_{D}$ for $C_{L}$.

For $N$,

$$
\begin{array}{r}
\frac{\partial N}{\partial r}=N\left[\frac{1}{\rho} \frac{d \rho}{d r}\left\{1+\frac{R e}{C_{M}} \frac{\partial C_{M}}{\partial R e}\right\}+\frac{1}{V^{2}} \frac{d w}{d r} \times\right. \\
\left\{\left(\frac{M}{C_{M}} \frac{\partial C_{M}}{\partial M}+\frac{R e}{C_{M}} \frac{\partial C_{M}}{\partial e}+2\right) \times\right. \\
\left.\left(v_{\theta}+w\right)+\frac{1}{C_{M}} \frac{\partial C_{M}}{\partial \alpha} v_{r}\right\}-
\end{array}
$$$$
\left.\frac{M}{C_{M}} \frac{\partial C_{M}}{\partial M} \frac{1}{a} \frac{d a}{d r}-\frac{R e}{C_{M}} \frac{\partial C_{M}}{\partial R e} \frac{1}{\mu} \frac{d \mu}{d r}\right]
$$$$
\frac{\partial N}{\partial v_{r}}=N \frac{v_{r}}{V^{2}}\left[\frac{M}{\bar{C}_{M}} \frac{\partial C_{M}}{\partial M}+\frac{R e}{C_{M}} \frac{\partial C_{M}}{\partial R}+\right.
$$$$
\left.2-\frac{1}{C_{M}} \frac{\partial C_{M}}{\partial \alpha} \frac{v_{\theta}+w}{v_{r}}\right]
$$$$
\frac{\partial N}{\partial v \theta}=N \frac{\left(v_{\theta}+w\right)}{V^{2}}\left[\frac{M}{C_{M}} \frac{\partial C_{M}}{\partial M}+\frac{R e}{C_{M}} \frac{\partial C_{M}}{\partial R e}+\right.
$$$$
\left.2+\frac{1}{C_{M}} \frac{\partial C_{M}}{\partial \alpha} \frac{v_{r}}{v_{\theta}+w}\right]
$$

$$
\begin{aligned}
& \frac{\partial N}{\partial \beta}=N \frac{1}{C_{M}} \frac{\partial C_{M}}{\partial \alpha} \\
& \frac{\partial N}{\partial \epsilon}=N \frac{1}{C_{M}} \frac{\partial C_{M}}{\partial \epsilon} \\
& \frac{\partial N}{\partial \rho}=N \frac{1}{\rho}\left[1+\frac{R e}{C_{M}} \frac{\partial C_{M}}{\partial R e}\right] \\
& \frac{\partial N}{\partial T}=-N\left[\frac{M}{C_{M}} \frac{\partial C_{M}}{\partial M} \frac{1}{2 T}+\frac{R e}{C_{M}} \frac{\partial C_{M}}{\partial R e} \frac{1}{\mu} \frac{\partial \mu}{\partial T}\right] \\
& \frac{\partial N}{\partial w}=\frac{\partial N}{\partial v_{\theta}} \\
& \frac{\partial N}{\partial I}=-\frac{N}{I}
\end{aligned}
$$

With these partial derivatives, the coefficients $a$ 's, $b$ 's, and $c$ 's can be easily calculated:

$$
\begin{aligned}
& a_{1}=\frac{\partial F}{\partial r}=\frac{\partial \Psi}{\partial r} \sin \beta+\frac{d w}{d r} \Lambda+(v \theta+w) \frac{\partial \Lambda}{\partial r}- \\
& v_{r} \frac{\partial \Delta}{\partial r}+\left(\frac{v_{\theta}}{r} \pm \Omega\right)^{2}-2 \frac{v_{\theta}}{r}\left(\frac{v_{\theta}}{r} \pm \Omega\right)+ \\
& 2 \frac{g}{r}\left(\frac{r_{0}}{r}\right)^{2} \\
& a_{2}=\frac{\partial F}{\partial \beta}=\Psi \cos \beta+\left(v_{\theta}+w\right) \frac{\partial \Lambda}{\partial \beta}-v_{r} \frac{\partial \Delta}{\partial \beta} \\
& a_{3}=\frac{\partial F}{\partial v_{r}}=\left(v_{\theta}+w\right) \frac{\partial \Lambda}{\partial v_{r}}-\Delta-v_{r} \frac{\partial \Delta}{\partial v_{r}} \\
& a_{4}=\frac{\partial F}{\partial v_{\theta}}=\Lambda+\left(v_{\theta}+w\right) \frac{\partial \Lambda}{\partial v_{\theta}}-v_{r} \frac{\partial \Delta}{\partial v_{\theta}}+2\left(\frac{v_{\theta}}{r} \pm \Omega\right) \\
& a_{5}=\frac{\partial F}{\partial \epsilon}=\left(v_{\theta}+w\right) \frac{\partial \Lambda}{\partial \epsilon}-v_{r} \frac{\partial \Delta}{\partial \epsilon} \\
& a_{6}=\frac{\partial F}{\partial \rho}=\left(v_{\theta}+w\right) \frac{\partial \Lambda}{\partial \rho}-v_{r} \frac{\partial \Delta}{\partial \rho} \\
& a_{7}=\frac{\partial F}{\partial T}=\left(v_{\theta}+w\right) \frac{\partial \Lambda}{\partial T}-v_{r} \frac{\partial \Delta}{\partial T} \\
& a_{8}=\frac{\partial F}{\partial w}=\Lambda+\left(v_{\theta}+w\right) \frac{\partial \Lambda}{\partial w}-v_{r} \frac{\partial \Delta}{\partial w} \\
& a_{\exists}=\frac{\partial F}{\partial W}=\frac{\partial \Psi}{\partial W} \sin \beta+\left(v_{\theta}+w\right) \frac{\partial \Lambda}{\partial W}-v_{r} \frac{\partial \Delta}{\partial W} \\
& b_{1}=\frac{\partial G}{\partial r}=\frac{\partial \Psi}{\partial r} \cos \beta-v_{r} \frac{\partial \Delta}{\partial r}-\frac{d w}{d r} \Delta- \\
& \left(v_{\theta}+w\right) \frac{\partial \Delta}{\partial r}+\frac{v_{r} v_{\theta}}{r^{2}} \\
& b_{2}=\frac{\partial G}{\partial \beta}=-\Psi \sin \beta-v_{r} \frac{\partial \Lambda}{\partial \beta}-\left(v_{\theta}+w\right) \frac{\partial \Delta}{\partial \beta}
\end{aligned}
$$




$$
\begin{aligned}
& b_{3}=\frac{\partial G}{\partial v_{r}}=-\Lambda-v_{r} \frac{\partial \Lambda}{\partial v_{r}}-\left(v_{\theta}+w\right) \frac{\partial \Delta}{\partial v_{r}}- \\
& 2\left(\frac{1}{2} \frac{v_{\theta}}{r} \pm \Omega\right) \\
& b_{4}=\frac{\partial G}{\partial v_{\theta}}=-v_{r} \frac{\partial \Lambda}{\partial v_{\theta}}-\Delta-\left(v_{\theta}+w\right) \frac{\partial \Delta}{\partial v_{\theta}}-\frac{v_{r}}{r} \\
& b_{5}=\frac{\partial G}{\partial \epsilon}=-v_{r} \frac{\partial \Lambda}{\partial \epsilon}-\left(v_{\theta}+w\right) \frac{\partial \Delta}{\partial \epsilon} \\
& b_{6}=\frac{\partial G}{\partial \rho}=-v_{r} \frac{\partial \Lambda}{\partial \rho}-\left(v_{\theta}+w\right) \frac{\partial \Delta}{\partial \rho} \\
& b_{7}=\frac{\partial G}{\partial T}=-v_{r} \frac{\partial \Lambda}{\partial T}-\left(v_{\theta}+w\right) \frac{\partial \Delta}{\partial T} \\
& b_{8}=\frac{\partial G}{\partial w}=-v_{r} \frac{\partial \Lambda}{\partial w}-\Delta-\left(v_{\theta}+w\right) \frac{\partial \Delta}{\partial w} \\
& b_{9}=\frac{\partial G}{\partial W}=\frac{\partial \Psi}{\partial \bar{W}} \cos \beta-v_{r} \frac{\partial \Lambda}{\partial \bar{W}}-\left(v_{\theta}+w\right) \frac{\partial \Delta}{\partial W} \\
& c_{1}=\frac{\partial H}{\partial r}=-\frac{1}{r^{2}}\left\{\Psi \cos \beta-v_{r} \Lambda-\left(v_{\theta}+w\right) \Delta-\right. \\
& \left.2 v_{r}\left(\frac{v_{\theta}}{r} \pm \Omega\right)\right\}+\frac{1}{r}\left\{\frac{\partial \Psi}{\partial r} \cos \beta-v_{r} \frac{\partial \Lambda}{\partial r}-\right. \\
& \left.\left(v_{\theta}+w\right) \frac{\partial \Delta}{\partial r}-\frac{d w}{d r} \Delta+2 \frac{v_{r} v_{\theta}}{r^{2}}\right\}+\frac{\partial N}{\partial r} \\
& c_{2}=\frac{\partial H}{\partial \beta}=\frac{1}{r}\left\{-\Psi \sin \beta-v_{r} \frac{\partial \Lambda}{\partial \beta}-\right. \\
& \left.\left(v_{\theta}+w\right) \frac{\partial \Delta}{\partial \beta}\right\}+\frac{\partial N}{\partial \beta} \\
& c_{3}=\frac{\partial H}{\partial v_{r}}=\frac{1}{r}\left\{-\Lambda-v_{r} \frac{\partial \Lambda}{\partial v_{r}}-\left(v_{\theta}+w\right) \frac{\partial \Delta}{\partial v_{r}}-\right. \\
& \left.2\left(\frac{v_{\theta}}{r} \pm \Omega\right)\right\}+\frac{\partial N}{\partial v_{r}} \\
& c_{4}=\frac{\partial H}{\partial v_{\theta}}=\frac{1}{r}\left\{-v_{r} \frac{\partial \Lambda}{\partial v_{\theta}}-\Delta-\left(v_{\theta}+w\right) \frac{\partial \Delta}{\partial v_{\theta}}-\right. \\
& \left.2 \frac{v_{r}}{r}\right\}+\frac{\partial N}{\partial v_{\theta}} \\
& c_{5}=\frac{\partial H}{\partial \epsilon}=\frac{1}{r}\left\{-v_{r} \frac{\partial \Lambda}{\partial \epsilon}-\left(v_{\theta}+w\right) \frac{\partial \Delta}{\partial \epsilon}\right\}+\frac{\partial N}{\partial \epsilon} \\
& c_{6}=\frac{\partial H}{\partial \rho}=\frac{1}{r}\left\{-v_{r} \frac{\partial \Lambda}{\partial \rho}-\left(v_{\theta}+w\right) \frac{\partial \Delta}{\partial \rho}\right\}+\frac{\partial N}{\partial \rho} \\
& c_{7}=\frac{\partial H}{\partial T^{\prime}}=\frac{1}{r}\left\{-v_{r} \frac{\partial \Lambda}{\partial T}-\left(v_{\theta}+w\right) \frac{\partial \Delta}{\partial T}\right\}+\frac{\partial N}{\partial T^{\prime}} \\
& c_{8}=\frac{\partial H}{\partial w}=\frac{1}{r}\left\{-v_{r} \frac{\partial \Lambda}{\partial w}-\Delta-\left(v_{\theta}+w\right) \frac{\partial \Delta}{\partial w}\right\}+\frac{\partial N}{\partial w} \\
& c_{9}=\frac{\partial H}{\partial W}=\frac{1}{r}\left\{\frac{\partial \Psi}{\partial W} \cos \beta-v_{r} \frac{\partial \Lambda}{\partial W}-\left(v_{\theta}+w\right) \frac{\partial \Delta}{\partial W}\right. \\
& c_{10}=\frac{\partial H}{\partial I}=\frac{\partial N}{\partial I}
\end{aligned}
$$

After the power cut-off, the thrust $f$ vanishes. Thus for $t>\vec{t}_{1}, \Psi$ and its derivatives are zero.

\section{References}

1 "Mathematical Theory of Rocket Flight," by J. B. Rosser, R. R. Newton, and G. L. Gross, MeGraw Hill Book Co., Inc., New York, 1947, particularly chapter III.

2 "The Perturbation Calculus in Missile Ballistics," by R. Drenick, Journal of the Franklin Institute, vol. 251, 1951, pp. 423436.

3 "Mathematics for Exterior Ballistics," by G. A. Bliss, John Wiley \& Sons, New York, 1944.

4 For possible tracking system, see "Pictorial Radio" by C. D. Tuska, Journal of the Franklin Institute, vol. 253, 1952, pp. 1-20; pp. 95-124. 